\title{
Anterior Segment Optical Coherence Tomography: Assisted Topographic Corneal Epithelial Thickness Distribution Imaging of a Keratoconus Patient
}

\author{
A. John Kanellopoulos ${ }^{a, b} \quad G^{2}$ eorge Asimellis ${ }^{a}$ \\ ${ }^{a}$ Laservision.gr Eye Institute, Athens, Greece; ${ }^{b}$ NYU Medical School, New York, N.Y., USA
}

\section{Key Words}

Anterior segment optical coherence tomography · Keratoconus · Epithelial imaging ·

Pentacam HR

\begin{abstract}
Purpose: To evaluate safety, efficacy and ease of measurement of epithelial thickness in a keratoconic patient based on anterior segment optical coherence tomography (AS-OCT). Methods: A 25-year-old male patient, previously diagnosed with keratoconus, with highly asymmetric manifestation among the two eyes, was subjected to AS-OCT corneal epithelial imaging. We investigated epithelial thickness and epithelial topographic thickness distribution. Results: Mean epithelial thickness was $51.97 \pm 0.70$ for the less affected right eye (OD), and $55.65 \pm 1.22$ for the more affected left eye (OS). Topographic epithelial thickness variability for the OD was $1.53 \pm 0.21 \mu \mathrm{m}$, while for the OS it was $9.80 \pm 0.41 \mu \mathrm{m}$. Conclusions: This case further supports our previous findings with high-frequency ultrasound measurements of the increase in overall epithelial thickness in keratoconic eyes in comparison with normal eyes. AS-OCT further offers ease of use and possibly higher predictability of measurement. This case report, based on AS-OCT imaging, verifies increased overall epithelial thickness in keratoconic eyes, as introduced by a previous study [Kanellopoulos et al.: Clin Ophthalmol 2012;6:789-800], based on high-frequency scanning ultrasound biomicroscopy imaging.
\end{abstract}




\section{Introduction}

We have introduced and reported [1] a potential novel clinical indicator for keratoconus (KCN) cornea instability assessment based on consistent, increased overall corneal epithelial thickness. We proposed this simple clinical parameter as an early keratectasia diagnostic factor. As in our previous report, most epithelial measurements have been performed in vivo, by high-frequency scanning ultrasound biomicroscopy (HF-UBM). We report herein similar data using anterior segment optical coherence tomography (AS-OCT).

\section{Case Report}

We present the case of a 25-year-old male subject, diagnosed with topographic and topometric asymmetrically manifested $\mathrm{KCN}$ among his two eyes. Informed consent was obtained from the subject.

The right eye (OD) had an uncorrected distance visual acuity of 20/20 and a manifest refraction of plano $-0.50 \times 170^{\circ}$. Corrected distance visual acuity with this prescription was $20 / 20+$. The left eye (OS), the more symptomatic one, had an uncorrected distance visual acuity of $20 / 80$ and a manifest refraction $-7.00-3.50 \times 75^{\circ}$. Corrected distance visual acuity with this prescription was $20 / 30$. The KCN diagnosis was further confirmed by corneal tomography imaging, obtained by WaveLight ${ }^{\circledR}$ Oculyzer ${ }^{\mathrm{TM}}$ II (Alcon Surgical, Ft. Worth, Tex., USA) which is a Pentacam HR (high-resolution) Scheimpflug imaging camera [2]. Examples of anterior corneal surface sagittal curvature maps are shown in fig. 1a for OD and fig. 1b for OS.

Four consecutive corneal scans in each eye were obtained by AS-OCT RTVue-100 (Optovue Inc., Fremont, Calif., USA), software version A6 $(9,0,27)$. Examples of OD and OS epithelial pachymetry maps (6 $\mathrm{mm}$ diameter) are provided in fig. 1c, d, respectively. Repeatability, which can be assessed by the standard deviation of the central epithelium of the 4 scans, was 0.60 and $1.50 \mu \mathrm{m}$ in OD and OS, respectively.

As shown in fig. 2a, OD had a central epithelial thickness (Epi CT) of $52.23 \pm 0.60 \mu \mathrm{m}$. Overall epithelial thickness (Epi Average) was computed by taking the average of the 17 segment thickness points shown in fig. 1c, d. The values displayed in each of the 17 segments are the average of the epithelial thickness measurements within each segment. OD had an Epi Average of $51.97 \pm 0.70 \mu \mathrm{m}$. OS had an Epi CT of $56.75 \pm 1.50 \mu \mathrm{m}$ and an Epi Average of $55.65 \pm 1.22 \mu \mathrm{m}$.

The epithelial topographic thickness variability (Epi Variability, fig. 2a) was calculated as the standard deviation of the 17 thickness points. Our results show that on average, the Epi Variability was $1.53 \pm 0.21 \mu \mathrm{m}$ for the less affected OD, while it was $9.80 \pm 0.41 \mu \mathrm{m}$ for the more affected OS.

\section{Discussion}

HF-UBM has known intrinsic examiner operative challenges (both for capture and interpretation), and although available for over a decade, it has seen very little clinical use and mainstream practice acceptance. Clinical availability of corneal epithelial mapping by ASOCT is currently in practice, and introduces a simple and effective clinical tool for corneal epithelial mapping. The data are very easily captured and epithelial thickness parameters 
Kanellopoulos et al.: Anterior Segment Optical Coherence Tomography: Assisted

Topographic Corneal Epithelial Thickness Distribution Imaging of a Keratoconus Patient

are automatically calculated by a novel software algorithm and presented in topographic imaging $[3,4]$.

This case illustrates a vivid agreement with previous findings by Kanellopoulos et al. [1] that an overall thicker epithelium with large variations can be observed in the topographically evident KCN eye, and to a much lesser degree in the less affected eye.

Qualitatively, the defining feature of the epithelium in KCN is the pattern, such that there is a thin epithelium over the cone surrounded by a thicker epithelium - as can be seen in fig. 1d. Quantitatively, epithelial thickness variations can be expressed as the topographic thickness variability and are computed by the standard deviation of the 17 segment thickness points. Specifically, in our case, it was $\pm 9.80 \mu \mathrm{m}$ in the affected KCN eye and \pm 1.53 $\mu \mathrm{m}$ in the less affected eye.

In fig. $2 \mathrm{~b}$, the graph compares the corresponding values of epithelial thickness from the HF-UBM study [1] and the case described herein. This case not only confirms our findings regarding the increased overall epithelial thickness in ectatic eyes, but also that the new ASOCT feature of epithelial imaging may illustrate these differences in a more pronounced way, as indicated by the data shown in fig. $2 \mathrm{a}, \mathrm{b}$.

\section{Conclusions}

The ease of use of AS-OCT epithelial imaging in comparison to HF-UBM as well as the increased predictability that may be offered by AS-OCT epithelial imaging may hold promise for wider clinical application, such as the screening of young adults for early KCN and, in a much wider perspective, potential candidates for laser cornea refractive surgery.

\section{Disclosure Statement}

Dr. Kanellopoulos is a consultant to Alcon WaveLight.

Mr. Asimellis has no financial interest in the devices presented herein.

\section{References}

1 Kanellopoulos AJ, Aslanides I, Asimellis G: Correlation between epithelial thickness in normal corneas, untreated ectatic corneas, and ectatic corneas previously treated with CXL; is overall epithelial thickness a very early ectasia prognostic factor? Clin Ophthalmol 2012;6:789-800.

-2 Kanellopoulos AJ, Asimellis G: Correlation between central corneal thickness, anterior chamber depth, and corneal keratometry as measured by Oculyzer II and WaveLight OB820 in preoperative cataract surgery patients. J Refract Surg 2012;22:1-6.

-3 Haque S, Simpson T, Jones L: Corneal and epithelial thickness in keratoconus: a comparison of ultrasonic pachymetry, Orbscan II, and optical coherence tomography. J Refract Surg 2006;22:486-493.

-4 Sin S, Simpson TL: The repeatability of corneal and corneal epithelial thickness measurements using optical coherence tomography. Optom Vis Sci 2006;83:360-365. 


\section{Case Reports in \\ Ophthalmology}

\begin{tabular}{l|l}
\hline \multicolumn{2}{l|}{ Case Rep Ophthalmol 2013;4:74-78 } \\
\hline DOI: $10.1159 / 000350630$ & $\begin{array}{l}\text { ○ 2013 S. Karger AG, Basel } \\
\text { www.karger.com/cop }\end{array}$ \\
\hline
\end{tabular}

Kanellopoulos et al.: Anterior Segment Optical Coherence Tomography: Assisted

Topographic Corneal Epithelial Thickness Distribution Imaging of a Keratoconus Patient

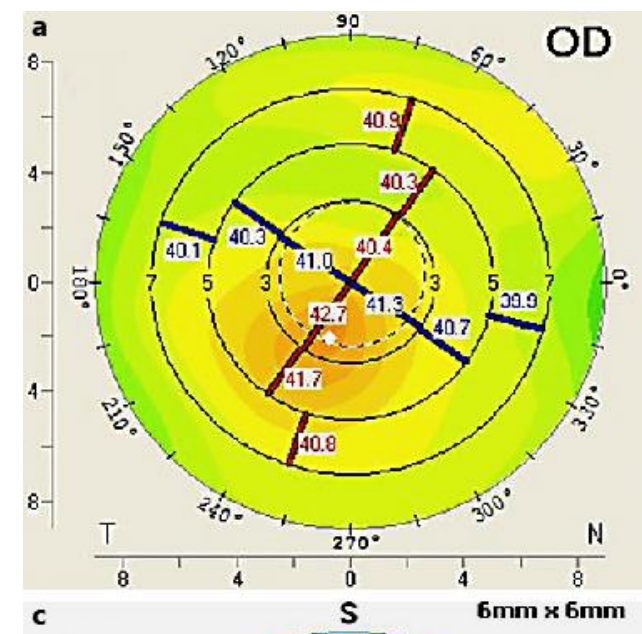

C

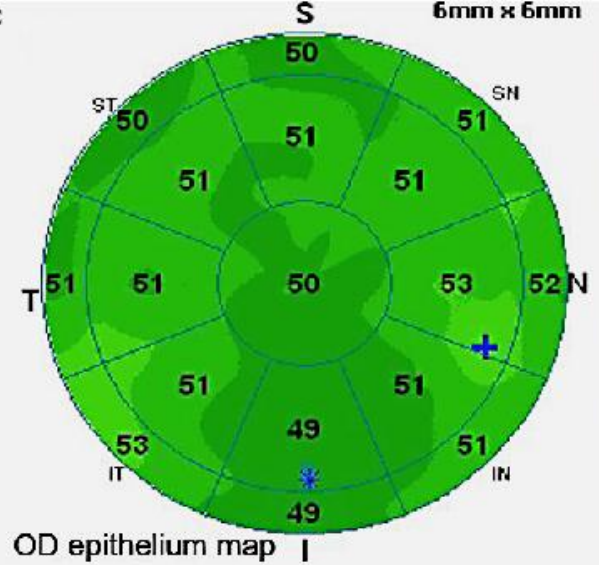

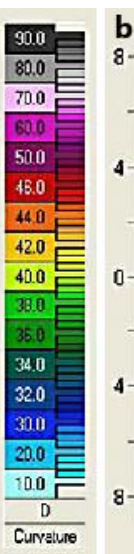
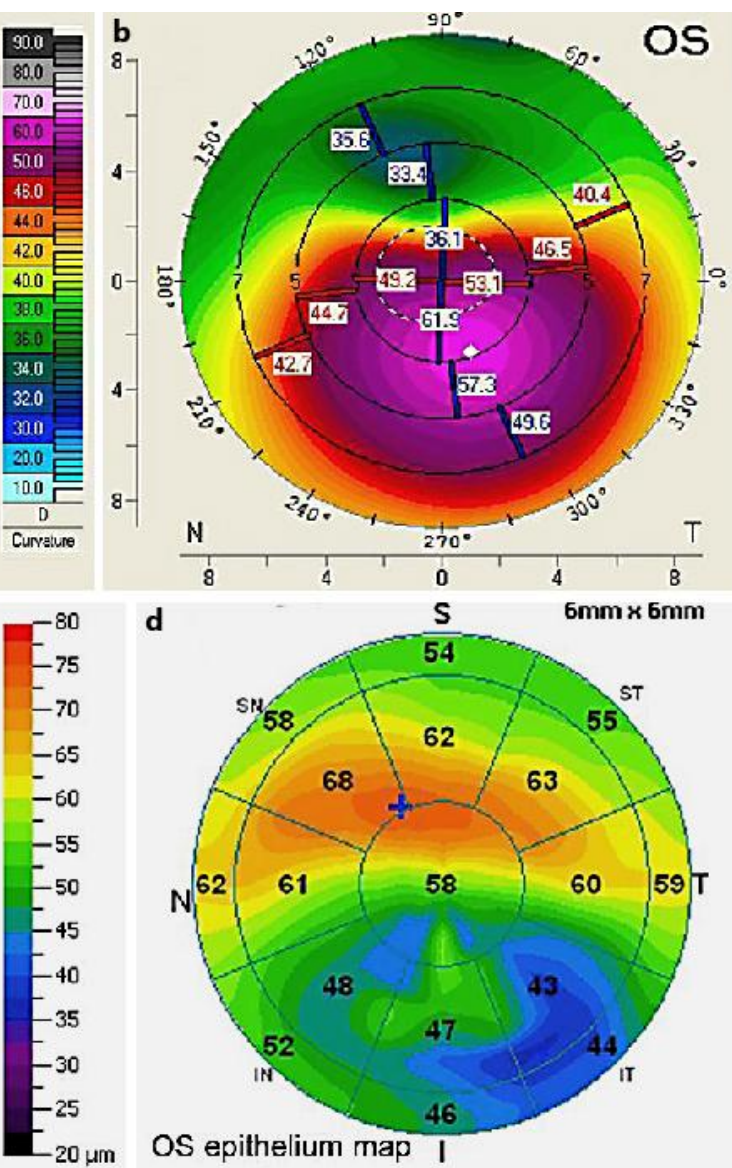

Fig. 1. a Tomographic anterior corneal sagittal curvature map for OD. b Tomographic anterior corneal sagittal curvature map for OS. c AS-OCT epithelial thickness map for OD. d AS-OCT epithelial thickness map for OS. 
Kanellopoulos et al.: Anterior Segment Optical Coherence Tomography: Assisted

Topographic Corneal Epithelial Thickness Distribution Imaging of a Keratoconus Patient

\begin{tabular}{|c|c|c|c|c|c|c|c|}
\hline a & CCT & Min CT & Epi CT & Epi min & Epi max & $\begin{array}{c}\text { Epl } \\
\text { Variability }\end{array}$ & $\begin{array}{c}\text { Epi } \\
\text { Average }\end{array}$ \\
\hline & & & Right & ye $(O D)$ & & & \\
\hline average & 557.00 & 538.33 & 52.23 & 48.00 & 53.67 & 1.53 & 51.97 \\
\hline stdev & 3.61 & 1.15 & 0.60 & 1.20 & 0.77 & 0.21 & 0.70 \\
\hline $\max$ & 560 & 539 & 53 & 49 & 55 & 1.70 & 52.80 \\
\hline $\min$ & 553 & 537 & 50 & 47 & 53 & 1.30 & 50.40 \\
\hline & & & Left & ye (OS) & & & \\
\hline average & 539.00 & 456.75 & 56.75 & 36.50 & 72.75 & 9.80 & 55.65 \\
\hline stdev & 5.48 & 5.19 & 1.50 & 1.73 & 0.96 & 0.41 & 1.22 \\
\hline $\max$ & 543 & 463 & 58 & 38 & 74 & 10.30 & 56.80 \\
\hline $\min$ & 531 & 452 & 55 & 35 & 72 & 9.30 & 54.60 \\
\hline
\end{tabular}

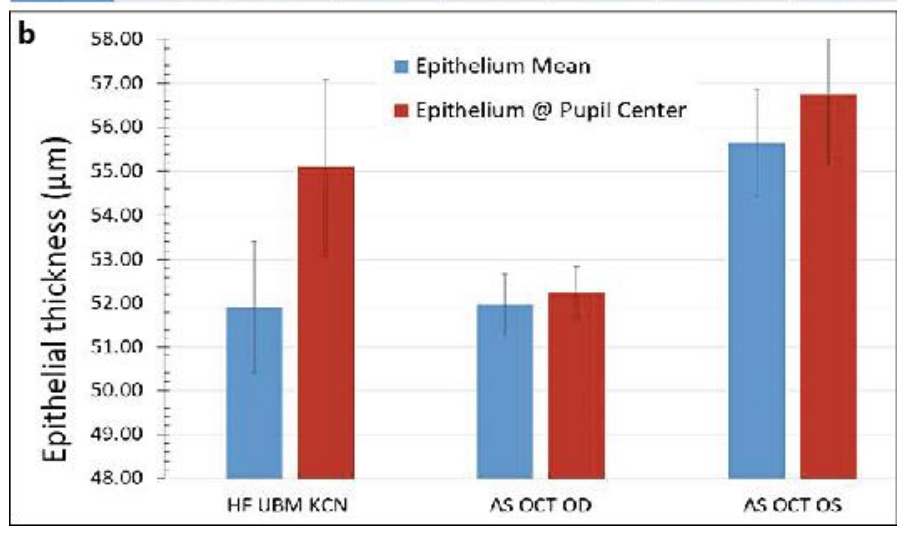

Fig. 2. a Corneal and epithelial thickness measurements for OD and OS. Values correspond to averages from 4 AS-OCT acquisitions. All values are in micrometers. Mean and standard deviations are calculated for the 4 scans. CCT = Central corneal thickness; Min = minimum; Max = maximum; stdev = standard deviation; Min CT = minimum corneal thickness; Epi min = epithelial minimum thickness; Epi max = epithelial maximum thickness. b Graph showing epithelial thickness averages for the KCN group, as reported by Kanellopoulos et al. [1] using HF-UBM, and the respective epithelial thicknesses for OD and OS as measured by AS-OCT. 